\title{
Some Properties of a Class of Harmonic Multivalent Functions Defined by an Integral Operator
}

\author{
Aqeel Ketab AL-khafaji ${ }^{a} \quad$ Waggas Galib Atshan $^{b} \quad$ Salwa Salman Abed $^{c}$ \\ ${ }^{a}$ Department of Mathematics, College of Education for Pure Sciences, University of Babylon, Babil, Iraq. \\ ${ }^{b}$ Department of Mathematics, College Of Computer Science \& Information Technology, University of Al- \\ Qadisiyah, Al-Qadisiyah, Iraq. \\ ${ }^{c}$ Department of Mathematics, College of Education for Pure Sciences - Ibn Al-Haytham, University of Baghdad., \\ Baghdad, Iraq. \\ aqeelketab@gmail.com_waggashnd@gmail.com_salwaalbundi@yahoo.com
}

Submission date:- 25/11/2018 Acceptance date:- 8/1/2019 Publication date:- 4/4/2019

Keywords: Harmonic multivalent function; coefficient inequality; linear operator; extreme points.

\section{Abstract}

Some properties of a class of harmonic multivalent functions defined by an integral operator are introduced, like, coefficient estimates, distortion property, extreme points, inclusion results and closure under an integral operator for this class are obtained.

\section{Introduction}

Let $u$ and $v$ are real harmonic functions in the simply connected domain $D \subseteq \mathbb{C}$, then the continuous functions $f=u+i v$, defined in $D$ is said to be harmonic in $D$. In any simply connected domain $D \subseteq \mathbb{C}$, we can write $f=h+\bar{g}$, where $\mathrm{h}$ and $\mathrm{g}$ are analytic in D. We call $h$ the analytic part and $g$ the co-analytic part of $f$.

A necessary and sufficient condition for $f$ to be locally univalent and sense preserving in $D$ is that $\left|h^{\prime}(z)\right|>$ $\left|g^{\prime}(z)\right| D$ ( see Clunie and Sheil-Small [1], see also [2]).

Denote by $H$ the class of functions $f=h+\bar{g}$ that are harmonic univalent and sense-preserving in the open unit $\operatorname{disc} \mathrm{U}=\{\mathrm{z}:|\mathrm{z}|<1\}$ where $h$ and $g$ are analytic in $D$ and $\mathrm{f}$ is normalized by $f(0)=h(0)=f_{z}(0)-1=0$.

The class $H_{p}(n)(p, n \in N=\{1,2,3, \ldots\})$, consisting of all p-valent harmonic functions $f=h+\bar{g}$ that are sense-preserving in $\mathrm{U}$ was defined by Ahuja and Jahangiri [3], where $h$ and $g$ are of the form

$$
h(z)=z^{p}+\sum_{k=2}^{\infty} a_{k+p-1} z^{k+p-1}, g(z)=\sum_{k=1}^{\infty} b_{k+p-1} z^{k+p-1},\left|b_{p}\right|<1 .
$$

It may be worth nothing that, when $p=1$ the class $H_{p}(n)$ was defined and studied by Jahangiri et al. [4]. In (1985) Salagean [5] introduced an integral operator $\mathrm{I}^{\mathrm{n}}$ and in (2009) Cotîrlă [6] introduced it in a slightly modified from as below

(1) $\mathrm{I}^{0} \mathrm{f}(\mathrm{z})=\mathrm{f}(\mathrm{z})$;

(2) $I^{1} f(z)=\operatorname{If}(z)=p \int_{0}^{z} f(t) t^{-1} d t ;$

(3) $I^{\mathrm{n}} \mathrm{f}(\mathrm{z})=\mathrm{I}\left(\mathrm{I}^{\mathrm{n}-1} \mathrm{f}(\mathrm{z})\right), \mathrm{n} \in \mathrm{N}, \mathrm{f} \in \mathrm{A}$, where $\mathrm{A}=\left\{\mathrm{f} \in \mathrm{H}: \mathrm{f}(\mathrm{z})=\mathrm{z}+\mathrm{a}_{2} \mathrm{z}^{2}+\cdots\right\}$ and $H=H(U)$, the class of analytic functions in $\mathrm{U}$.

The modified Salagean integral operator [7] of $f=h+\bar{g}$ given by (1) is defined as

where

$$
I^{n} f(z)=I^{n} h(z)+(-1)^{n} \overline{I^{n} g(z)}
$$

and

$$
I^{n} h(z)=z^{p}+\sum_{k=2}^{\infty}\left(\frac{p}{k+p-1}\right)^{n} a_{k+p-1} z^{k+p-1}
$$

$$
I^{n} g(z)=\sum_{k=1}^{\infty}\left(\frac{p}{k+p-1}\right)^{n} b_{k+p-1} z^{k+p-1}
$$

Now, we define the class of harmonic multivalent functions as follows:

Let $H_{p}(n, \mu, \gamma, \delta)$ we denote the class of harmonic multivalent functions of the form (1) such that

$$
\begin{aligned}
& \operatorname{Re}\left\{\frac{I^{\mathrm{n}} f(z)+2 \delta z^{p}}{(1-\gamma) z^{p}+\gamma I^{n+1} f(z)}\right\}>\mu, \\
& \quad \text { where } \mathrm{I}^{\mathrm{n}} \text { is defined by (2), } 0 \leq \gamma, \mu<1,0 \leq \delta<\frac{1}{2}, \mathrm{n}, \mathrm{k}, \mathrm{p} \in \mathrm{N}, \mathrm{z} \in \mathrm{U} .
\end{aligned}
$$


Let $\overline{H_{p}}(n, \mu, \gamma, \delta)$ we denote the subclass of $\mathrm{H}_{\mathrm{p}}(\mathrm{n}, \mu, \gamma, \delta)$ consisting of harmonic multivalent functions $f_{n}=h+$ $\overline{g_{n}}$ so that $\mathrm{h}$ and $\mathrm{g}_{\mathrm{n}}$ are of the form:

$$
h(z)=z^{p}-\sum_{k=2}^{\infty} a_{k+p-1} z^{k+p-1}, g(z)=(-1)^{n-1} \sum_{k=1}^{\infty} b_{k+p-1} z^{k+p-1},
$$

where $a_{k+p-1} \geq 0, b_{k+p-1} \geq 0$ and $\left|b_{p}\right|<1$.

We deep it worthwhile to point here the relevance of the function class $\overline{H_{p}}(n, \mu, \gamma, \delta)$ with those classes of functions which have been studied recently. So we note that

(i) If we take $\delta=0$, then the class $\overline{H_{p}}(n, \mu, \gamma, \delta)$ reduced to the class $\overline{H_{p}}(n, \beta, t)$ [5] of course after replace $\mu$ and $\gamma$ by $\beta$, t respectively.

(ii) The class $\overline{\mathrm{H}_{\mathrm{p}}}(\mathrm{n}, \mu, \gamma, \delta)$ reduced to the class $\overline{H_{p}}(n, \beta)$ [7], if we take $\delta=0$ and $\gamma=1$.

(iii) Also our class $\overline{H_{p}}(n, \mu, \gamma, \delta)$ reduced to the class $\overline{H_{p}}(n+1, n, \alpha, 0)$ [6] with values $\delta=0, \gamma=1$ and $\mu=\beta$.

In this paper, we study a class of harmonic multivalent functions defined by an integral operator. Coefficient bounds, distortion bounds, extreme points, inclusion results and closure under an integral operator for functions in the class $\overline{H_{p}}(n, \mu, \gamma, \delta)$ are obtained.

Lemma1.1: [8] Let $\alpha \geq 0$. Then $\operatorname{Re}(w)>\alpha$ if and only if $|w-(1+\alpha)|<|w+(1-\alpha)|$, where $w$ be any complex number.

\section{Main Results}

In our first theorem, we give the sufficient coefficient condition for harmonic functions belonging to the class $\mathrm{H}_{\mathrm{p}}(\mathrm{n}, \mu, \gamma, \delta)$.

Theorem 2.1: Let $f=h+\bar{g}$ be given by (1). If

where

$$
\sum_{k=2}^{\infty} \varphi(n, p, k, \mu, \gamma, \delta)\left|a_{k+p-1}\right|+\sum_{k=1}^{\infty} \Phi(n, p, k, \mu, \gamma, \delta)\left|b_{k+p-1}\right| \leq 1,
$$

$$
\begin{gathered}
\varphi(n, p, k, \mu, \gamma, \delta)=\frac{\left(\frac{p}{k+p-1}\right)^{n}\left[1-\mu \gamma\left(\frac{p}{k+p-1}\right)\right]}{(1+\delta-\mu)} \\
\Phi(n, p, k, \mu, \gamma, \delta)=\frac{\left(\frac{p}{k+p-1}\right)^{n}\left[1+\mu \gamma\left(\frac{p}{k+p-1}\right)\right]}{(1+\delta-\mu)}, \\
0 \leq \gamma, \mu<1, \quad 0 \leq \delta<\frac{1}{2}, \quad n, k, p \in N .
\end{gathered}
$$

Then $f$ is harmonic multivalent sense - preservyngin $U$ and $f \in H_{p}(n, \mu, \gamma, \delta)$.

Proof. Denote $\left(\frac{\mathrm{p}}{\mathrm{k}+\mathrm{p}-1}\right)$ by $\rho$ through in this proof. To prove that $f \in H_{p}(n, \mu, \gamma, \delta)$ by the condition (3), we only need to show that if (5) holds, then

$$
\operatorname{Re}\left\{\frac{\mathrm{I}^{\mathrm{n}} \mathrm{f}(\mathrm{z})+2 \delta \mathrm{z}^{\mathrm{p}}}{(1-\gamma) \mathrm{z}^{\mathrm{p}}+\gamma \mathrm{I}^{\mathrm{n}+1} \mathrm{f}(\mathrm{z})}\right\}=\operatorname{Re} \frac{\mathrm{L}(\mathrm{z})}{\mathrm{K}(\mathrm{z})} \geq \mu,
$$

where $\mathrm{z}=\mathrm{re}^{\mathrm{i} \theta}, 0 \leq \theta \leq 2 \pi, 0 \leq \mathrm{r}<1$, and $0 \leq \mu<1$.

Note that

By using Lemma 1.1, it suffices to show that

$$
\begin{aligned}
& \mathrm{L}(\mathrm{z})=\mathrm{I}^{\mathrm{n}} \mathrm{f}(\mathrm{z})+2 \delta \mathrm{z}^{\mathrm{p}} \quad \text { and } \\
& \mathrm{K}(\mathrm{z})=(1-\gamma) \mathrm{z}^{\mathrm{p}}+\gamma \mathrm{I}^{\mathrm{n}+1} \mathrm{f}(\mathrm{z}) .
\end{aligned}
$$

Substituting $L(z)$ and $K(z)$ in (6), we obtain

$$
|L(z)+(1-\mu) K(z)|-|L(z)-(1+\mu) K(z)| \geq 0 .
$$

$|\mathrm{L}(\mathrm{z})+(1-\mu) \mathrm{K}(\mathrm{z})|-|\mathrm{L}(\mathrm{z})-(1+\mu) \mathrm{K}(\mathrm{z})|$

$$
=\left|I^{\mathrm{n}} \mathrm{f}(\mathrm{z})+2 \delta \mathrm{z}^{\mathrm{p}}+(1-\mu)\left[(1-\gamma) \mathrm{z}^{\mathrm{p}}+\gamma \mathrm{I}^{\mathrm{n}+1} \mathrm{f}(\mathrm{z})\right]\right|-\left|\mathrm{I}^{\mathrm{n}} \mathrm{f}(\mathrm{z})+\delta \mathrm{z}^{\mathrm{p}}-(1+\mu)\left[(1-\gamma) \mathrm{z}^{\mathrm{p}}+\gamma \mathrm{I}^{\mathrm{n}+1} \mathrm{f}(\mathrm{z})\right]\right|
$$




$$
\begin{aligned}
& =\mid z^{p}+\sum_{k=2}^{\infty} \rho^{n} a_{k+p-1} z^{k+p-1}+(-1)^{n} \sum_{k=1}^{\infty} \rho^{n} \overline{b_{k+p-1} z^{k+p-1}}+2 \delta z^{p} \\
& +(1-\mu)\left[(1-\gamma) \mathrm{z}^{\mathrm{p}}+\gamma \mathrm{z}^{\mathrm{p}}\right. \\
& \left.+\gamma \sum_{\mathrm{k}=2}^{\infty} \rho^{\mathrm{n}+1} \mathrm{a}_{\mathrm{k}+\mathrm{p}-1} \mathrm{z}^{\mathrm{k}+\mathrm{p}-1}+\gamma(-1)^{\mathrm{n}+1} \sum_{\mathrm{k}=1}^{\infty} \rho^{\mathrm{n}+1} \overline{\mathrm{b}_{\mathrm{k}+\mathrm{p}-1} \mathrm{z}^{\mathrm{k}+\mathrm{p}-1}}\right] \mid \\
& -\mid \mathrm{z}^{\mathrm{p}} \\
& +\sum_{\mathrm{k}=2}^{\infty} \rho^{\mathrm{n}} \mathrm{a}_{\mathrm{k}+\mathrm{p}-1} \mathrm{z}^{\mathrm{k}+\mathrm{p}-1}+(-1)^{\mathrm{n}} \sum_{\mathrm{k}=1}^{\infty} \rho^{\mathrm{n}} \overline{\mathrm{b}_{\mathrm{k}+\mathrm{p}-1} \mathrm{z}^{\mathrm{k}+\mathrm{p}-1}}+\delta \mathrm{z}^{\mathrm{p}} \\
& -(1+\mu)\left[(1-\gamma) z^{p}+\gamma z^{p}\right. \\
& \left.+\gamma \sum_{\mathrm{k}=2}^{\infty} \rho^{\mathrm{n}+1} \mathrm{a}_{\mathrm{k}+\mathrm{p}-1} \mathrm{z}^{\mathrm{k}+\mathrm{p}-1}+\gamma(-1)^{\mathrm{n}+1} \sum_{\mathrm{k}=1}^{\infty} \rho^{\mathrm{n}+1} \overline{\mathrm{b}_{\mathrm{k}+\mathrm{p}-1} \mathrm{z}^{\mathrm{k}+\mathrm{p}-1}}\right] \mid \\
& =\mid(2+2 \delta-\mu) \mathrm{z}^{\mathrm{p}} \\
& +\sum_{\mathrm{k}=2}^{\infty} \rho^{\mathrm{n}}[1+(1-\mu) \gamma \rho] \mathrm{a}_{\mathrm{k}+\mathrm{p}-1} \mathrm{z}^{\mathrm{k}+\mathrm{p}-1}-(-1)^{\mathrm{n}+1} \sum_{\mathrm{k}=1}^{\infty} \rho^{\mathrm{n}}[1-(1-\mu) \gamma \rho] \overline{\mathrm{b}_{\mathrm{k}+\mathrm{p}-1} \mathrm{z}^{\mathrm{k}+\mathrm{p}-1}} \\
& -\mid \mu z^{p} \\
& +\sum_{\mathrm{k}=2}^{\infty} \rho^{\mathrm{n}}[1-(1+\mu) \gamma \rho] \mathrm{a}_{\mathrm{k}+\mathrm{p}-1} \mathrm{z}^{\mathrm{k}+\mathrm{p}-1}-(-1)^{\mathrm{n}+1} \sum_{\mathrm{k}=1}^{\infty} \rho^{\mathrm{n}}[1+(1+\mu) \gamma \rho] \overline{\mathrm{b}_{\mathrm{k}+\mathrm{p}-1} \mathrm{z}^{\mathrm{k}+\mathrm{p}-1}} \mid \\
& \geq(2+2 \delta-\mu)|\mathrm{z}|^{\mathrm{p}}-\sum_{\mathrm{k}=2}^{\infty} \rho^{\mathrm{n}}[1+(1-\mu) \gamma \rho]\left|\mathrm{a}_{\mathrm{k}+\mathrm{p}-1}\right||\mathrm{z}|^{\mathrm{k}+\mathrm{p}-1}-\sum_{\mathrm{k}=1}^{\infty} \rho^{\mathrm{n}}[1-(1-\mu) \gamma \rho]\left|\mathrm{b}_{\mathrm{k}+\mathrm{p}-1}\right||\mathrm{z}|^{\mathrm{k}+\mathrm{p}-1}-\mu|\mathrm{z}|^{\mathrm{p}} \\
& -\sum_{\mathrm{k}=2}^{\infty} \rho^{\mathrm{n}}[1-(1+\mu) \gamma \rho]\left|\mathrm{a}_{\mathrm{k}+\mathrm{p}-1}\right||\mathrm{z}|^{\mathrm{k}+\mathrm{p}-1}-\sum_{\mathrm{k}=1}^{\infty} \rho^{\mathrm{n}}[1+(1+\mu) \gamma \rho]\left|\mathrm{b}_{\mathrm{k}+\mathrm{p}-1}\right||\mathrm{z}|^{\mathrm{k}+\mathrm{p}-1} \\
& =2(1+\delta-\mu)|\mathrm{z}|^{\mathrm{p}}-\sum_{\mathrm{k}=2}^{\infty} \rho^{\mathrm{n}}[1+(1-\mu) \gamma \rho+1-(1+\mu) \gamma \rho]\left|\mathrm{a}_{\mathrm{k}+\mathrm{p}-1}\right||\mathrm{z}|^{\mathrm{k}+\mathrm{p}-1} \\
& -\sum_{\mathrm{k}=1}^{\infty} \rho^{\mathrm{n}}[1-(1-\mu) \gamma \rho+1+(1+\mu) \gamma \rho]\left|\mathrm{b}_{\mathrm{k}+\mathrm{p}-1}\right||\mathrm{z}|^{\mathrm{k}+\mathrm{p}-1} \\
& =2(1+\delta-\mu)|\mathrm{z}|^{\mathrm{p}}-\sum_{\mathrm{k}=2}^{\infty} 2 \rho^{\mathrm{n}}[1-\mu \gamma \rho]\left|\mathrm{a}_{\mathrm{k}+\mathrm{p}-1}\right||\mathrm{z}|^{\mathrm{k}+\mathrm{p}-1}-\sum_{\mathrm{k}=1}^{\infty} 2 \rho^{\mathrm{n}}[1+\mu \gamma \rho]\left|\mathrm{b}_{\mathrm{k}+\mathrm{p}-1}\right||\mathrm{z}|^{\mathrm{k}+\mathrm{p}-1} \\
& =2(1+\delta-\mu)|\mathrm{z}|^{\mathrm{p}}\left[1-\left\{\sum_{\mathrm{k}=2}^{\infty} \frac{\rho^{\mathrm{n}}[1-\mu \gamma \rho]}{(1+\delta-\mu)}\left|\mathrm{a}_{\mathrm{k}+\mathrm{p}-1}\right||\mathrm{z}|^{\mathrm{k}+\mathrm{p}-1}+\sum_{\mathrm{k}=1}^{\infty} \frac{2 \rho^{\mathrm{n}}[1+\mu \gamma \rho]}{(1+\delta-\mu)}\left|\mathrm{b}_{\mathrm{k}+\mathrm{p}-1}\right||\mathrm{z}|^{\mathrm{k}+\mathrm{p}-1}\right\}\right] \\
& =2(1+\delta-\mu)\left[1-\left\{\sum_{\mathrm{k}=2}^{\infty} \frac{\rho^{\mathrm{n}}[1-\mu \gamma \rho]}{(1+\delta-\mu)}\left|\mathrm{a}_{\mathrm{k}+\mathrm{p}-1}\right|+\sum_{\mathrm{k}=1}^{\infty} \frac{2 \rho^{\mathrm{n}}[1+\mu \gamma \rho]}{(1+\delta-\mu)}\left|\mathrm{b}_{\mathrm{k}+\mathrm{p}-1}\right|\right\}\right] . \\
& |\mathrm{L}(\mathrm{z})+(1-\mu) \mathrm{K}(\mathrm{z})|-|\mathrm{L}(\mathrm{z})-(1+\mu) \mathrm{K}(\mathrm{z})| \\
& =2(1+\delta-\mu)[1 \\
& -\left\{\sum_{\mathrm{k}=2}^{\infty} \frac{\left(\frac{\mathrm{p}}{\mathrm{k}+\mathrm{p}-1}\right) \mathrm{n}\left[1-\mu \gamma\left(\frac{\mathrm{p}}{\mathrm{k}+\mathrm{p}-1}\right)\right]}{(1+\delta-\mu)}\left|\mathrm{a}_{\mathrm{k}+\mathrm{p}-1}\right|\right. \\
& \left.\left.+\sum_{\mathrm{k}=1}^{\infty} \frac{\left(\frac{\mathrm{p}}{\mathrm{k}+\mathrm{p}-1}\right) \mathrm{n}\left[1+\mu \gamma\left(\frac{\mathrm{p}}{\mathrm{k}+\mathrm{p}-1}\right)\right]}{(1+\delta-\mu)}\left|\mathrm{b}_{\mathrm{k}+\mathrm{p}-1}\right|\right\}\right] \geq 0,
\end{aligned}
$$


by (5), which implies that $f \in H_{p}(n, \mu, \gamma, \delta)$.

The harmonic multivalent function

$$
f(z)=z^{p}+\sum_{k=2}^{\infty} \frac{1}{\varphi(n, p, k \cdot \mu, \gamma, \delta)} x_{k} z^{k+p-1}+\sum_{k=1}^{\infty} \frac{1}{\Phi(n, p, k \cdot \mu, \gamma, \delta)} y_{k} z^{k+p-1},
$$

where $\mathrm{n} \in \mathrm{N}$ and $\sum_{\mathrm{k}=2}^{\infty} \mathrm{x}_{\mathrm{k}}+\sum_{\mathrm{k}=1}^{\infty} \mathrm{y}_{\mathrm{k}}=1$, shows that the coefficient bound given by (5) is sharp. The functions of the form (7) are in the class $H_{p}(n, \mu, \gamma, \delta)$, because

$$
\begin{gathered}
\sum_{\mathrm{k}=2}^{\infty} \varphi(\mathrm{n}, \mathrm{p}, \mathrm{k}, \mu, \gamma, \delta)\left|\mathrm{a}_{\mathrm{k}+\mathrm{p}-1}\right|+\sum_{\mathrm{k}=1}^{\infty} \Phi(\mathrm{n}, \mathrm{p}, \mathrm{k}, \mu, \gamma, \delta)\left|\mathrm{b}_{\mathrm{k}+\mathrm{p}-1}\right| \\
=\sum_{\mathrm{k}=2}^{\infty} \varphi(\mathrm{n}, \mathrm{p}, \mathrm{k}, \mu, \gamma, \delta) \frac{1}{\varphi(\mathrm{n}, \mathrm{p}, \mathrm{k}, \mu, \gamma, \delta)}\left|\mathrm{x}_{\mathrm{k}}\right|+\sum_{\mathrm{k}=1}^{\infty} \Phi(\mathrm{n}, \mathrm{p}, \mathrm{k}, \mu, \gamma, \delta) \frac{1}{\Phi(\mathrm{n}, \mathrm{p}, \mathrm{k}, \mu, \gamma, \delta)}\left|\mathrm{y}_{\mathrm{k}}\right| \\
=\sum_{\mathrm{k}=2}^{\infty}\left|\mathrm{x}_{\mathrm{k}}\right|+\sum_{\mathrm{k}=1}^{\infty}\left|\mathrm{y}_{\mathrm{k}}\right|=1 .
\end{gathered}
$$

Now, we show that the condition (5) is also necessary for the function $f_{n}=h+\overline{g_{n}}$ to belong to $\overline{H_{p}}(n, \mu, \gamma, \delta)$, where $h$ and $g_{n}$ are given by (4).

Theorem 2. 2: Let $f_{n}=h+\overline{g_{n}}$, be given by (4). Then $f_{n} \in \overline{H_{p}}(n, \mu, \gamma, \delta)$ if and only if

$$
\sum_{k=2}^{\infty} \varphi(n, p, k, \mu, \gamma, \delta) a_{k+p-1}+\sum_{k=1}^{\infty} \Phi(n, p, k . \mu, \gamma, \delta) b_{k+p-1} \leq 1,
$$

where $0 \leq \gamma, \mu<1,0 \leq \delta<\frac{1}{2}, n \in N$, with $b_{k+p-1}>a_{k+p-1}$, for every $k \geq 2$.

Proof. Denote $\left(\frac{p}{k+p-1}\right)$ by $\rho$ through in this proof. Since $\overline{H_{p}}(n, \mu, \gamma, \delta) \subset H_{p}(n, \mu, \gamma, \delta)$, we need only to prove the " only if " of the theorem. For functions $f_{n}$ of the form (4), we notice that the condition

is equivalent to

$$
\operatorname{Re}\left\{\frac{I^{n} f(z)+2 \delta z^{p}}{(1-\gamma) z^{p}+\gamma I^{n+1} f(z)}\right\}>\mu,
$$

$$
\operatorname{Re}\left\{\frac{-\sum_{k=2}^{\infty} \rho^{n}[1-\mu \gamma \rho] a_{k+p-1} z^{k+p-1}+(-1)^{2 n-1} \sum_{k=1}^{\infty} \rho^{n}[1+\mu \gamma \rho] b_{k+p-1} \bar{z}^{k+p-1}}{z^{p}-\gamma \sum_{k=2}^{\infty} \rho^{n+1} a_{k+p-1} z^{k+p-1}+\gamma(-1)^{2 n} \sum_{k=1}^{\infty} \rho^{n+1} b_{k+p-1} \bar{z}^{k+p-1}}\right\}
$$

$$
\geq 0 \text {. }
$$

Now, the last inequality (9) must hold for all values of $\mathrm{z}$ in U. Choosing the value of $\mathrm{z}$ on the positive real axis where $0 \leq \mathrm{z}=\mathrm{r}<1$, we have $\mathrm{b}_{\mathrm{k}+\mathrm{p}-1}>\mathrm{a}_{\mathrm{k}+\mathrm{p}-1}$, for every $\mathrm{k} \geq 2$,

$$
\frac{\left[-\sum_{\mathrm{k}=2}^{\infty} \rho^{\mathrm{n}}[1-\mu \gamma \rho] \mathrm{a}_{\mathrm{k}+\mathrm{p}-1} \mathrm{r}^{\mathrm{k}+\mathrm{p}-1}-\sum_{\mathrm{k}=1}^{\infty} \rho^{\mathrm{n}}[1+\mu \gamma \rho] \mathrm{b}_{\mathrm{k}+\mathrm{p}-1} \mathrm{r}^{\mathrm{k}+\mathrm{p}-1}\right]}{\left[1-\gamma \sum_{\mathrm{k}=2}^{\infty} \rho^{\mathrm{n}+1} \mathrm{a}_{\mathrm{k}+\mathrm{p}-1} \mathrm{r}^{\mathrm{k}+\mathrm{p}-1}+\gamma(-1)^{2 \mathrm{n}} \sum_{\mathrm{k}=1}^{\infty} \rho^{\mathrm{n}+1} \mathrm{~b}_{\mathrm{k}+\mathrm{p}-1} \mathrm{r}^{\mathrm{k}+\mathrm{p}-1}\right]} \geq 0 .
$$

We notice that the expression in (10) is negative for $r$ sufficiently close to 1 , when the condition (8) does not hold. Hence there exist $z_{0}=r_{0}$ in $(0,1)$ for which the quotient in (10) is negative. This contradicts the required condition for $f_{n} \in \overline{H_{p}}(n, \mu, \gamma, \delta)$

\section{Extreme Points and Distortion Bounds.}

Here, we consider the extreme points of closed convex hull of $\overline{H_{p}}(n, \mu, \gamma, \delta)$, denoted by $\operatorname{clco} \overline{H_{p}}(n, \mu, \gamma, \delta)$ and we obtain the distortion bounds for functions in $\overline{H_{p}}(n, \mu, \gamma, \delta)$.

Theorem 3.1: Let $f_{n}$ given by (4). Then $f_{n} \in \operatorname{clco} \overline{H_{p}}(n, \mu, \gamma, \delta)$ if and only if

$$
\begin{gathered}
f_{n}(z)=\sum_{k=1}^{\infty}\left[x_{k+p-1} h_{k+p-1}(z)+y_{k+p-1} g_{k+p-1}(z)\right], \\
\text { where } h_{p}(z)=z^{p}, h_{k+p-1}(z)=z^{p}-\frac{1}{\varphi(n, p, k, \mu, \gamma, \delta)} z^{k+p-1}, k \geq 2,3, \ldots, \text { and } \\
g_{k+p-1}(z)=z^{p}+(-1)^{n-1} \frac{1}{\Phi(n, p, k, \mu, \gamma, \delta)} z^{k+p-1}, \quad k \geq 2,3, \ldots \\
x_{k+p-1} \geq 0, \quad y_{k+p-1} \geq 0, x_{p}=1-\sum_{k=2}^{\infty} x_{k+p-1}-\sum_{k=1}^{\infty} y_{k+p-1} .
\end{gathered}
$$

In particular, the extreme points of $\overline{H_{p}}(n, \mu, \gamma, \delta)$ are $\left\{h_{k+p-1}\right\}$ and $\left\{g_{k+p-1}\right\}$.

Proof. Suppose

$$
f_{n}(z)=\sum_{k=1}^{\infty}\left[x_{k+p-1} h_{k+p-1}(z)+y_{k+p-1} g_{k+p-1}(z)\right]
$$


On the other hand

$$
\begin{gathered}
=\sum_{\mathrm{k}=1}^{\infty}\left(\mathrm{x}_{\mathrm{k}+\mathrm{p}-1}+\mathrm{y}_{\mathrm{k}+\mathrm{p}-1}\right) \mathrm{z}^{\mathrm{p}}-\sum_{\mathrm{k}=2}^{\infty} \frac{1}{\varphi(\mathrm{n}, \mathrm{p}, \mathrm{k}, \mu, \gamma, \delta)} \mathrm{x}_{\mathrm{k}+\mathrm{p}-1} \mathrm{z}^{\mathrm{k}+\mathrm{p}-1} \\
+(-1)^{\mathrm{n}-1} \sum_{\mathrm{k}=1}^{\infty} \frac{1}{\Phi(\mathrm{n}, \mathrm{p}, \mathrm{k}, \mu, \gamma, \delta)} \mathrm{y}_{\mathrm{k}+\mathrm{p}-1} \mathrm{z}^{\mathrm{k}+\mathrm{p}-1} \\
=\mathrm{z}^{\mathrm{p}}-\sum_{\mathrm{k}=2}^{\infty} \frac{1}{\varphi(\mathrm{n}, \mathrm{p}, \mathrm{k}, \mu, \gamma, \delta)} \mathrm{x}_{\mathrm{k}+\mathrm{p}-1} \mathrm{z}^{\mathrm{k}+\mathrm{p}-1}+(-1)^{\mathrm{n}-1} \sum_{\mathrm{k}=1}^{\infty} \frac{1}{\Phi(\mathrm{n}, \mathrm{p}, \mathrm{k}, \mu, \gamma, \delta)} \mathrm{y}_{\mathrm{k}+\mathrm{p}-1} \mathrm{z}^{\mathrm{k}+\mathrm{p}-1} .
\end{gathered}
$$

$$
\begin{gathered}
\sum_{\mathrm{k}=2}^{\infty} \varphi(\mathrm{n}, \mathrm{p}, \mathrm{k}, \mu, \gamma, \delta)\left|\mathrm{a}_{\mathrm{k}+\mathrm{p}-1}\right|+\sum_{\mathrm{k}=1}^{\infty} \Phi(\mathrm{n}, \mathrm{p}, \mathrm{k}, \mu, \gamma, \delta)\left|\mathrm{b}_{\mathrm{k}+\mathrm{p}-1}\right| \\
=\sum_{\mathrm{k}=2}^{\infty} \varphi(\mathrm{n}, \mathrm{p}, \mathrm{k}, \mu, \gamma, \delta)\left(\frac{1}{\varphi(\mathrm{n}, \mathrm{p}, \mathrm{k}, \mu, \gamma, \delta)} \mathrm{x}_{\mathrm{k}+\mathrm{p}-1}\right)+\sum_{\mathrm{k}=1}^{\infty} \Phi(\mathrm{n}, \mathrm{p}, \mathrm{k}, \mu, \gamma, \delta)\left(\frac{1}{\Phi(\mathrm{n}, \mathrm{p}, \mathrm{k}, \mu, \gamma, \delta)} \mathrm{y}_{\mathrm{k}+\mathrm{p}-1}\right) \\
=\sum_{\mathrm{k}=2}^{\infty} \mathrm{x}_{\mathrm{k}+\mathrm{p}-1}+\sum_{\mathrm{k}=1}^{\infty} \mathrm{y}_{\mathrm{k}+\mathrm{p}-1}=1-\mathrm{x}_{\mathrm{p}} \leq 1 .
\end{gathered}
$$

Therefore $f_{n}(z) \in \operatorname{clco} \overline{H_{p}}(n, \mu, \gamma, \delta)$.

Conversely, if $f_{n}(z) \in \operatorname{clco} \overline{H_{p}}(n, \mu, \gamma, \delta)$. Assume

Set $\quad x_{k+p-1}=\varphi(n, p, k, \mu, \gamma, \delta) a_{k+p-1}, k=2,3, \ldots \quad$ and

$$
x_{p}=1-\sum_{k=2}^{\infty} x_{k+p-1}-\sum_{k=1}^{\infty} y_{k+p-1} .
$$

Now , consider

$$
\begin{aligned}
& f_{n}(\mathrm{z})=z^{p}-\sum_{k=2}^{\infty} a_{k+p-1} z^{k+p-1}+(-1)^{n-1} \sum_{k=1}^{\infty} b_{k+p-1}, \overline{z^{k+p-1}} \\
& =\mathrm{z}^{\mathrm{p}}-\sum_{\mathrm{k}=2}^{\infty} \frac{1}{\varphi(\mathrm{n}, \mathrm{p}, \mathrm{k}, \mu, \gamma, \delta)} \mathrm{x}_{\mathrm{k}+\mathrm{p}-1} \mathrm{z}^{\mathrm{k}+\mathrm{p}-1}+(-1)^{\mathrm{n}-1} \sum_{\mathrm{k}=1}^{\infty} \frac{1}{\Phi(\mathrm{n}, \mathrm{p}, \mathrm{k}, \mu, \gamma, \delta)} \mathrm{y}_{\mathrm{k}+\mathrm{p}-1} \overline{z^{k+p-1}} \\
& =\mathrm{z}^{\mathrm{p}}-\sum_{\mathrm{k}=2}^{\infty}\left[\mathrm{z}^{\mathrm{p}}-\mathrm{h}_{\mathrm{k}+\mathrm{p}-1}(\mathrm{z})\right] \mathrm{x}_{\mathrm{k}+\mathrm{p}-1}+\sum_{\mathrm{k}=1}^{\infty}\left[\mathrm{z}^{\mathrm{p}}-\mathrm{g}_{\mathrm{k}+\mathrm{p}-1}(\mathrm{z})\right] \mathrm{y}_{\mathrm{k}+\mathrm{p}-1} \\
& =\left[1-\sum_{\mathrm{k}=2}^{\infty} \mathrm{x}_{\mathrm{k}+\mathrm{p}-1}-\sum_{\mathrm{k}=1}^{\infty} \mathrm{y}_{\mathrm{k}+\mathrm{p}-1}\right] \mathrm{z}^{\mathrm{p}}+\sum_{\mathrm{k}=2}^{\infty} \mathrm{x}_{\mathrm{k}+\mathrm{p}-1} \mathrm{~h}_{\mathrm{k}+\mathrm{p}-1}(\mathrm{z})+\sum_{\mathrm{k}=2}^{\infty} \mathrm{x}_{\mathrm{k}+\mathrm{p}-1} \mathrm{~h}_{\mathrm{k}+\mathrm{p}-1}(\mathrm{z}) \\
& =\sum_{\mathrm{k}+\mathrm{p}-1} \mathrm{~h}_{\mathrm{k}+\mathrm{p}-1}(\mathrm{z})+\sum_{\mathrm{k}=1}^{\infty} \mathrm{x}_{\mathrm{k}+\mathrm{p}-1} \mathrm{~h}_{\mathrm{k}+\mathrm{p}-1}(\mathrm{z})
\end{aligned}
$$

Theorem 3.2: Let $f_{n} \in \overline{H_{p}}(n, \mu, \gamma, \delta)$. Then for $|z|=r<1$, we have

$$
\begin{array}{ll}
\text { and } & \left|f_{n}(z)\right| \leq(1+b) r^{p}+\{\psi(n, p, k, \mu, \gamma, \delta)-\phi(n, p, k, \mu, \gamma, \delta)\} r^{p+1}, \\
\text { where } & \left|f_{n}(z)\right| \geq(1+b) r^{p}-\{\psi(n, p, k, \mu, \gamma, \delta)+\phi(n, p, k, \mu, \gamma, \delta)\} r^{p+1},
\end{array}
$$

$$
\begin{gathered}
\psi(n, p, k, \mu, \gamma, \delta)=\frac{(1+\delta-\mu \gamma)}{\left(\frac{p}{p+1}\right)^{n}\left[1-\mu \gamma\left(\frac{p}{p+1}\right)\right]} \\
\phi(n, p, k, \mu, \gamma, \delta)=\frac{(1+\delta+\mu \gamma)}{\left(\frac{p}{p+1}\right)^{n}\left[1-\mu \gamma\left(\frac{p}{p+1}\right)\right]} .
\end{gathered}
$$

Proof. We prove the right hand side from (11) for $\left|f_{n}\right|$. The proof of inequality (12) is similar. Let $f_{n} \in$ $\overline{H_{p}}(n, \mu, \gamma, \delta)$. Taking the absolute value of $f_{n}$, therefore by Theorem (2.2), we obtain :

$$
\begin{gathered}
\left|f_{n}(z)\right|=\left|z^{p}-\sum_{k=2}^{\infty} a_{k+p-1} z^{k+p-1}+(-1)^{n-1} \sum_{k=1}^{\infty} b_{k+p-1} \overline{z^{k+p-1}}\right| \\
\leq \mathrm{r}^{\mathrm{p}}-\sum_{\mathrm{k}=2}^{\infty} \mathrm{a}_{\mathrm{k}+\mathrm{p}-1} \mathrm{r}^{\mathrm{k}+\mathrm{p}-1}+\sum_{\mathrm{k}=1}^{\infty} \mathrm{b}_{\mathrm{k}+\mathrm{p}-1} \mathrm{r}^{\mathrm{k}+\mathrm{p}-1} \\
=\mathrm{r}^{\mathrm{p}}+\mathrm{b}_{\mathrm{p}} \mathrm{r}^{\mathrm{p}}+\psi(\mathrm{n}, \mathrm{p}, 2, \mu, \gamma, \delta) \sum_{\mathrm{k}=2}^{\infty} \frac{1}{\psi(\mathrm{n}, \mathrm{p}, \mathrm{k}, \mu, \gamma, \delta)}\left(\mathrm{a}_{\mathrm{k}+\mathrm{p}-1}+\mathrm{b}_{\mathrm{k}+\mathrm{p}-1}\right) \mathrm{r}^{\mathrm{k}+\mathrm{p}-1} \\
\leq\left(1+\mathrm{b}_{\mathrm{p}}\right) \mathrm{r}^{\mathrm{p}}+\psi(\mathrm{n}, \mathrm{p}, 2, \mu, \gamma, \delta) \mathrm{r}^{\mathrm{p}+1}
\end{gathered}
$$




$$
\begin{aligned}
& {\left[\sum_{\mathrm{k}=2}^{\infty} \varphi(\mathrm{n}, \mathrm{p}, \mathrm{k}, \mu, \gamma, \delta) \mathrm{a}_{\mathrm{k}+\mathrm{p}-1}+\Phi(\mathrm{n}, \mathrm{p}, \mathrm{k}, \mu, \gamma, \delta) \mathrm{b}_{\mathrm{k}+\mathrm{p}-1}\right]} \\
& \leq(1+\mathrm{b}) \mathrm{r}^{\mathrm{p}}+\{\psi(\mathrm{n}, \mathrm{p}, \mathrm{k}, \mu, \gamma, \delta)-\phi(\mathrm{n}, \mathrm{p}, \mathrm{k}, \mu, \gamma, \delta)\} \mathrm{r}^{\mathrm{p}+1}
\end{aligned}
$$

\section{Convolution Property and Convex Combination of the Class $\overline{H_{p}}(n, \mu, \gamma, \delta)$.}

Here, we want to prove two theorems, the first theorem about convolution for the class $\overline{H_{p}}(n, \mu, \gamma, \delta)$ and in the second theorem, we prove that the class $\overline{H_{p}}(n, \mu, \gamma, \delta)$ is closed under convex combination.

The convolution of two harmonic functions

and

$$
f_{n}(z)=z^{p}-\sum_{k=2}^{\infty} a_{k+p-1} z^{k+p-1}+(-1)^{n-1} \sum_{k=1}^{\infty} b_{k+p-1}(\bar{z})^{k+p-1},
$$

is defined as

$$
q_{n}(z)=z^{p}-\sum_{k=2}^{\infty} c_{k+p-1} z^{k+p-1}+(-1)^{n-1} \sum_{k=1}^{\infty} d_{k+p-1}(\bar{z})^{k+p-1},
$$

$$
\begin{aligned}
& \left(f_{n} * q_{n}\right)(z)=f_{n}(z) * q_{n}(z) \\
= & \mathrm{z}^{\mathrm{p}}-\sum_{\mathrm{k}=2}^{\infty} \mathrm{a}_{\mathrm{k}+\mathrm{p}-1} \mathrm{c}_{\mathrm{k}+\mathrm{p}-1} \mathrm{z}^{\mathrm{k}+\mathrm{p}-1}+(-1)^{\mathrm{n}-1} \sum_{\mathrm{k}=1}^{\infty} \mathrm{b}_{\mathrm{k}+\mathrm{p}-1} \mathrm{~d}_{\mathrm{k}+\mathrm{p}-1}(\overline{\mathrm{z}})^{\mathrm{k}+\mathrm{p}-1},
\end{aligned}
$$

Using this definition, we show that the class $\overline{\mathrm{H}_{\mathrm{p}}}(\mathrm{n}, \mu, \gamma, \delta)$ is closed under convolution.

Theorem 4.1: For $0 \leq \tau \leq \mu<1,0 \leq \gamma<1,0 \leq \delta<\frac{1}{2}$, let $f_{n} \in \overline{H_{p}}(n, \mu, \gamma, \delta)$ and $q_{n} \in \overline{H_{p}}(n, \tau, \gamma, \delta)$. Then

Proof. Let

$$
f_{n} * q_{n} \in \overline{H_{p}}(n, \mu, \gamma, \delta) \subset \overline{H_{p}}(n, \tau, \gamma, \delta)
$$

be in the class $\overline{H_{p}}(n, \mu, \gamma, \delta)$ and

$$
f_{n}(z)=z^{p}-\sum_{k=2}^{\infty} a_{k+p-1} z^{k+p-1}+(-1)^{n-1} \sum_{k=1}^{\infty} b_{k+p-1}(\overline{\mathrm{z}})^{\mathrm{k}+\mathrm{p}-1}
$$

be in $\overline{H_{p}}(n, \tau, \gamma, \delta)$.

$$
q_{n}(z)=z^{p}-\sum_{k=2}^{\infty} c_{k+p-1} z^{k+p-1}+(-1)^{n-1} \sum_{k=1}^{\infty} d_{k+p-1}(\bar{z})^{k+p-1}
$$

Then the convolution $f_{n} * q_{n}$ is given by (15), we want to show that the coefficients of $f_{n} * q_{n}$ satisfies the required condition given in Theorem (2.2).

For $q_{n} \in \overline{H_{p}}(n, \tau, \gamma, \delta)$, we note that $c_{k+p-1}<1$ and $d_{k+p-1}<1$. Now consider convolution functions $f_{n} * q_{n}$ as follows:

$$
\begin{aligned}
\sum_{\mathrm{k}=2}^{\infty} \varphi(\mathrm{n}, \mathrm{p}, \mathrm{k}, \tau, \gamma, \delta) \mathrm{a}_{\mathrm{k}+\mathrm{p}-1} \mathrm{c}_{\mathrm{k}+\mathrm{p}-1}+\sum_{\mathrm{k}=1}^{\infty} \Phi(\mathrm{n}, \mathrm{p}, \mathrm{k}, \tau, \gamma, \delta) \mathrm{b}_{\mathrm{k}+\mathrm{p}-1} \mathrm{~d}_{\mathrm{k}+\mathrm{p}-1} \\
\leq \sum_{\mathrm{k}=2}^{\infty} \varphi(\mathrm{n}, \mathrm{p}, \mathrm{k}, \tau, \gamma, \delta) \mathrm{a}_{\mathrm{k}+\mathrm{p}-1}+\sum_{\mathrm{k}=1}^{\infty} \Phi(\mathrm{n}, \mathrm{p}, \mathrm{k}, \tau, \gamma, \delta) \mathrm{b}_{\mathrm{k}+\mathrm{p}-1} \\
\leq \sum_{\mathrm{k}=2}^{\infty} \varphi(\mathrm{n}, \mathrm{p}, \mathrm{k}, \mu, \gamma, \delta) \mathrm{a}_{\mathrm{k}+\mathrm{p}-1}+\sum_{\mathrm{k}=1}^{\infty} \Phi(\mathrm{n}, \mathrm{p}, \mathrm{k}, \mu, \gamma, \delta) \mathrm{b}_{\mathrm{k}+\mathrm{p}-1}
\end{aligned}
$$

$\leq 1 . \quad\left(\right.$ since $0 \leq \tau \leq \mu<1$ and $\left.f_{n} \in \overline{H_{p}}(n, \mu, \gamma, \delta)\right)$.

Now, we prove the second theorem in this section as follows

Theorem 4.2: The class $\overline{H_{p}}(n, \mu, \gamma, \delta)$ is closed under convex combinations.

Proof. For $\mathrm{s}=1,2, \ldots$. Suppose that $f_{n, S} \in \overline{H_{p}}(n, \mu, \gamma, \delta)$, where $f_{n, S}$ is given by

$$
f_{n, s}(z)=z^{p}-\sum_{k=2}^{\infty} a_{s, k+p-1} z^{k+p-1}+(-1)^{n-1} \sum_{k=1}^{\infty} b_{s, k+p-1}(\bar{z})^{k+p-1} \text {. }
$$

Then by Theorem 2.2, we have

$$
\sum_{\mathrm{k}=2}^{\infty} \varphi(\mathrm{n}, \mathrm{p}, \mathrm{k}, \mu, \gamma, \delta) \mathrm{a}_{\mathrm{s}, \mathrm{k}+\mathrm{p}-1}+\sum_{\mathrm{k}=1}^{\infty} \Phi(\mathrm{n}, \mathrm{p}, \mathrm{k}, \mu, \gamma, \delta) \mathrm{b}_{\mathrm{s}, \mathrm{k}+\mathrm{p}-1} \leq 1,
$$

For $\sum_{s=1}^{\infty} \mathrm{t}_{\mathrm{s}}=1, \quad 0 \leq \mathrm{t}_{\mathrm{s}} \leq 1$.

Then the convex combination of $f_{n, s}$ written as 
$\sum_{s=1}^{\infty} t_{s} f_{n, s}(z)=z^{p}-\sum_{k=2}^{\infty}\left(\sum_{s=1}^{\infty} t_{s} a_{s, k+p-1}\right) z^{k+p-1}+(-1)^{n-1} \sum_{k=1}^{\infty}\left(\sum_{s=1}^{\infty} t_{s} b_{s, k+p-1}\right)(\bar{z})^{k+p-1}$.

Now , by using the inequality (16), we obtain

$\sum_{\mathrm{k}=2}^{\infty} \varphi(\mathrm{n}, \mathrm{p}, \mathrm{k}, \mu, \gamma, \delta)\left(\sum_{s=1}^{\infty} \mathrm{t}_{\mathrm{s}} \mathrm{a}_{\mathrm{s}, \mathrm{k}+\mathrm{p}-1}\right)+\sum_{\mathrm{k}=1}^{\infty} \Phi(\mathrm{n}, \mathrm{p}, \mathrm{k}, \mu, \gamma, \delta)\left(\sum_{s=1}^{\infty} \mathrm{t}_{\mathrm{s}} \mathrm{b}_{\mathrm{s}, \mathrm{k}+\mathrm{p}-1}\right)$

$=\sum_{\mathrm{s}=1}^{\infty} \mathrm{t}_{\mathrm{s}}\left(\sum_{\mathrm{k}=2}^{\infty} \varphi(\mathrm{n}, \mathrm{p}, \mathrm{k}, \mu, \gamma, \delta) \mathrm{a}_{\mathrm{s}, \mathrm{k}+\mathrm{p}-1}+\sum_{\mathrm{k}=1}^{\infty} \Phi(\mathrm{n}, \mathrm{p}, \mathrm{k}, \mu, \gamma, \delta) \mathrm{b}_{\mathrm{s}, \mathrm{k}+\mathrm{p}-1}\right)$

$\leq \sum_{s=1}^{\infty} t_{s}=1, \quad$ which is the required coefficient condition

Next, we introduce the last theorem in this paper of the class $\overline{H_{p}}(n, \mu, \gamma, \delta)$ under the generalized BernardiLibera-Livingston integral operator (see [9] and [10]) $\mathcal{F}_{u}(f)$ can be defined as

$$
\mathcal{F}_{u}(f)=\frac{u+p}{z^{u}} \int_{0}^{z} t^{u-1} f(t) d t, \quad u>-1 .
$$

Theorem 4.3: Let $f_{n}(z) \in \overline{H_{p}}(n, \mu, \gamma, \delta)$. Then

$$
\mathcal{F}_{u}\left(f_{n}(z)\right) \in \overline{H_{p}}(n, \mu, \gamma, \delta)
$$

Proof. From definition of $\mathcal{F}_{u}\left(f_{n}(z)\right)$ given by (17), it follows that

$$
\begin{aligned}
\mathcal{F}_{\mathrm{u}}\left(f_{n}(\mathrm{z})\right) & =\frac{\mathrm{u}+\mathrm{p}}{\mathrm{z}^{\mathrm{u}}} \int_{0}^{\mathrm{z}} \mathrm{t}^{\mathrm{u}-1}\left[\mathrm{t}^{\mathrm{p}}-\sum_{\mathrm{k}=2}^{\infty} \mathrm{a}_{\mathrm{k}+\mathrm{p}-1} \mathrm{t}^{\mathrm{k}+\mathrm{p}-1}+(-1)^{\mathrm{n}-1} \sum_{\mathrm{k}=1}^{\infty} \mathrm{b}_{\mathrm{k}+\mathrm{p}-1} \mathrm{t}^{\mathrm{k}+\mathrm{p}-1}\right] \mathrm{dt} \\
& =\mathrm{z}^{\mathrm{p}}-\sum_{\mathrm{k}=2}^{\infty} \frac{\mathrm{u}+\mathrm{p}}{\mathrm{u}+\mathrm{p}+\mathrm{k}-1} \mathrm{a}_{\mathrm{k}+\mathrm{p}-1} \mathrm{z}^{\mathrm{k}+\mathrm{p}-1}+(-1)^{\mathrm{n}-1} \sum_{\mathrm{k}=1}^{\infty} \frac{\mathrm{u}+\mathrm{p}}{\mathrm{u}+\mathrm{p}+\mathrm{k}-1} \mathrm{~b}_{\mathrm{k}+\mathrm{p}-1}(\bar{z})^{k+p-1} \\
& =\mathrm{z}^{\mathrm{p}}-\sum_{\mathrm{k}=2}^{\infty} \mathrm{G}_{\mathrm{k}+\mathrm{p}-1} \mathrm{z}^{\mathrm{k}+\mathrm{p}-1}+(-1)^{\mathrm{n}-1} \sum_{\mathrm{k}=1}^{\infty} \mathrm{L}_{\mathrm{k}+\mathrm{p}-1}(\bar{z})^{k+p-1}
\end{aligned}
$$

where

$$
\begin{aligned}
\mathrm{G}_{\mathrm{k}+\mathrm{p}-1} & =\frac{\mathrm{u}+\mathrm{p}}{\mathrm{u}+\mathrm{p}+\mathrm{k}-1} \mathrm{a}_{\mathrm{k}+\mathrm{p}-1}, \text { and } \\
\mathrm{L}_{\mathrm{k}+\mathrm{p}-1} & =\frac{\mathrm{u}+\mathrm{p}}{\mathrm{u}+\mathrm{p}+\mathrm{k}-1} \mathrm{~b}_{\mathrm{k}+\mathrm{p}-1} .
\end{aligned}
$$

Hence

$\sum_{\mathrm{k}=2}^{\infty} \varphi(\mathrm{n}, \mathrm{p}, \mathrm{k}, \mu, \gamma, \delta) \frac{\mathrm{u}+\mathrm{p}}{\mathrm{u}+\mathrm{p}+\mathrm{k}-1} \mathrm{a}_{\mathrm{k}+\mathrm{p}-1}+\sum_{\mathrm{k}=1}^{\infty} \Phi(\mathrm{n}, \mathrm{p}, \mathrm{k} \cdot \mu, \gamma, \delta) \frac{\mathrm{u}+\mathrm{p}}{\mathrm{u}+\mathrm{p}+\mathrm{k}-1} \mathrm{~b}_{\mathrm{k}+\mathrm{p}-1}$

$\leq \sum_{\mathrm{k}=2}^{\infty} \varphi(\mathrm{n}, \mathrm{p}, \mathrm{k}, \mu, \gamma, \delta) \mathrm{a}_{\mathrm{k}+\mathrm{p}-1}+\sum_{\mathrm{k}=1}^{\infty} \Phi(\mathrm{n}, \mathrm{p}, \mathrm{k}, \mu, \gamma, \delta) \mathrm{b}_{\mathrm{k}+\mathrm{p}-1}$

$\leq 1$. (by 8 )

Therefore by Theorem (2.2), we have $\mathcal{F}_{\mathrm{u}}\left(\mathrm{f}_{\mathrm{n}}(\mathrm{z})\right) \in \overline{H_{p}}(n, \mu, \gamma, \delta)$

\section{Acknowledgement}

The authors are thankful to the referee for his valuable comments and observations.

\section{CONFLICT OF INTERESTS}

There are no conflicts of interest.

\section{References}

1. J. Clunie and T. Sheil-Small, "Harmonic Univalent Functions", Ann. Acad. Sci. Fenn., Ser. A.I., Math., 9, 3-25. 1984

2. AL-khafaji, A. K.; Atshan, W. G.; Abed, S. S.. "On the Generalization of a Class of Harmonic Univalent Functions Defined by Differential Operator." Mathematics Vol. 6, no.12, 312. 2018.

3. J. M. Jahangiri and O. P. Ahuja, "Multivalent Harmonic Starlike Functions", Ann. Univ. Maric. CurieSklodowska Sect. A, 55 no.1. 1-13. 2001

4. J. M. Jahangiri, Y. C. Kim and H. M. Srivastava, Construction of a Certain Class of Harmonic Close-toConvex Function Associated with Alexander Integral Transform, Int. Trans, And Special Func., 14(3), 237-242. 2003.

5. G. S. Salagean, "Subclass of Univalent Functions", Lecture Notes In Math., Springer-Verlag, 1013, pp,362372. 1983.

6. L. I. Cotîrlă," Harmonic Multivalent Functions Defined by Integral Operator" Studia Univ.Babes Bolyai Mathematica, Liv (1) pp, 65-74. 2009. 
7. R. Ezhilarasi, T. V. Sudharsan and K.G. Subramanian, "A Class of Harmonic Multivalent Functions Defined by an Integral Operator" Gen. Math. Notes, 22(1), 17-30. 2014.

8. Aqlan E. S. "Some Problem Connected with Geometric Function Theory" Ph. D. Thesis, Pune University, Pune. India 2004.

9. S. D. Bernardi, Convex and Starlike Univalent Function, Trans Amer. Math. Soc 135(1969),429-446.

10. Libera R. J. "Some Classes of Regular Univalent Functions" Proc. Amer. Math. Soc., 16, 755-758. 1965.

في هذ البحث قدمنا دراسة حول صنف من الدو ال التو اققية المتعددة التكافؤ

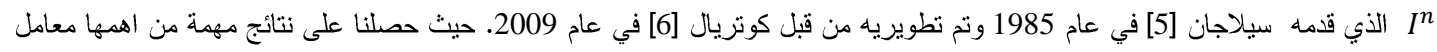
التقدير للصنف المتطرفة كذلك, بالإضافة الى العديد من النتائج الدذكورة في البحث. الكلمات الدالة: وظيفة متناسقة متعددة. عدم المساو اة معامل. عامل خطي النقاط القصوى. 